\title{
Rate effect and coupled evolution of atomic motions and potential landscapes
}

\author{
Pan Xiao · Jun Wang · Rong Yang · Fu-Jiu Ke · Meng-Fen Xia · Yi-Long Bai
}

Received: 14 March 2012 / Revised: 26 April 2013 / Accepted: 15 May 2013

(C)The Chinese Society of Theoretical and Applied Mechanics and Springer-Verlag Berlin Heidelberg 2013

\begin{abstract}
Since rate effect of materials plays a key role in impact engineering, the microscopic mechanism of rate effect is investigated at molecular level in this paper. The results show that rate effect on the strength of atomic system is closely related to the coupled evolution of atomic motions and potential landscapes. Accordingly, it becomes possible to develop a new algorithm of molecular simulation, which could properly and efficiently demonstrate strain rate effect under a wide range of loading rates and unveil the mechanisms underlying the strain rate effects.
\end{abstract}

Keywords Strain rate effect - Atomic motions - Potential landscape $\cdot$ Molecular simulation

\section{Introduction}

The rate effect of materials under impact loadings, namely drastic increase of yield stress and strength of materials under strain rates beyond about $10^{3} \mathrm{~s}^{-1}$, is a key feature in impact and shock dynamics as well as a long standing problem in material science [1]. It is reported that rate effects exist in a wide range of materials [2-5]. Strain rates in most experiments range from $10^{-5}$ to $10^{7} \mathrm{~s}^{-1}$; and rate effect mainly emerges in plastic deformation and fracture, e.g., yield stress, flow stress, strength and fracture toughness $[3,6,7]$.

\footnotetext{
The project was supported by the National Basic Research Program of China (973 Program) (2012CB937500), the National Natural Science Foundation of China (11202212, 10932011, 11021262, 11172024, 11172305, and 11232013).
}

P. Xiao · J. Wang · R. Yang · M.-F. Xia · Y.-L. Bai (四)

State Key Laboratory of Nonlinear Mechanics (LNM), Institute of Mechanics, Chinese Academy of Sciences, 100190 Beijing, China

e-mail: baiyl@lnm.imech.ac.cn

\section{F.-J. Ke}

School of Physics and Nuclear Energy Engineering,

Beihang University, 100191 Beijing, China
However strain rate effects do not present themselves in a simple linear way. A distinct transition has been observed in the relationship of flow stress and strain rate in a number of metals, like copper, iron, etc. This indicates that there might be different mechanisms which can govern the rate effect $[1,3]$. So far, several mechanisms have been proposed, like dislocation mobility $[3,8]$, shock controlled mechanism of dislocation or twinning $[1,9,10]$, etc. All these mechanisms are very important in the understanding of the phenomena related to the rate effect. However, since most of them are phenomenological explanations, a microscopic or molecular understanding of the rate effect will be elusive.

Molecular dynamics (MD), with its ability to trace the dynamic process of each molecule, has become an orthodox method in elucidating microscopic mechanisms of mechanical behaviour of materials. Lots of MD simulations have been carried out to investigate the strain rate effect of nanoscale structures [11-14]. These simulations demonstrate some results similar to that observed in experiments. However the simulated sample sizes are restricted to tens of $\mathrm{nm}$, much smaller than that in laboratory tests. Moreover, the strain rates involved in previous MD simulations are quite high (usually higher than $10^{8} \mathrm{~s}^{-1}[11,13]$ in contrast to those appearing in most laboratory tests, like $10^{-3} \mathrm{~s}^{-1}$. The limitations of MD simulation should be attributed to its intrinsic length and time scales involved in the potential functions used to characterize the inter-molecular interactions [15]. Take the Lennard-Jones (L-J) potential,

$\phi=4 \varepsilon_{0}\left[\left(\frac{r_{0}}{r}\right)^{12}-\left(\frac{r_{0}}{r}\right)^{6}\right]$

as an example [16], there are 2 parameters, the intrinsic energy $\varepsilon_{0}$ and length $r_{0}$. Plus the molecular mass $m$, the intrinsic time scale (oscillating period of a molecule) in MD can be determined as $t_{\mathrm{MD}}=\sqrt{m r_{0}^{2} / \varepsilon_{0}}$, with a typical value of about $10^{-13} \mathrm{~s}$. To keep differential accuracy, the time step in MD should be much smaller than $t_{\mathrm{MD}}$, e.g., several fs or even shorter. As a result, MD simulations have to be restricted to a very short duration or extremely high strain rate. Therefore, 
it leads to a practical challenge when a larger system should be examined with a lower strain rate. Another challenge encountered is how the strain rate effect comes from Newton equation under potential filed in MD simulations

$m \frac{\mathrm{d}^{2} \boldsymbol{X}}{\mathrm{d} t^{2}}=-\nabla \phi(\boldsymbol{X})$

where $\boldsymbol{X}$ and $\phi$ denote molecular position and potential field, respectively. Note that there is no explicit dissipation term in the governing equation, then how the rate effect comes from.

On the other hand, comparing with full dynamics, quasi-static mechanical processes can be regarded as the other extreme. In this regard, we have proposed a molecular statistical thermodynamics (MST) method, which is designed for quasi-static analysis of molecular systems at finite temperature $[17,18]$. By representing the molecular thermal vibrations with local harmonic approximation [19], MST greatly releases the restriction induced by the time scales involved in MD simulations. Applications of MST to several nanomaterials have shown its advantage over MD with about hundred times higher efficiency [18,20,21]. Filling the gap between MST and MD techniques may lead to a new algorithm which can handle a wide range of strain rates.

In this work, molecular simulations are carried out to analyze molecular systems under a wide range of tensile strain rates. Here the focus is on the microscopic mechanism underlying the strain rate effect. Based on the understandings, alternative numerical algorithms are proposed in order to simulate molecular systems at finite temperature and under various strain rates efficiently. Numerical results and efficiency of the new algorithms are discussed in details.

\section{Two extremes of molecular simulations}

In order to clarify the essence of rate effect at atomic level and the foundation of the new algorithm, we firstly look at two extremes of molecular simulations: MD and MST.

For the rate effect analysis, a one-dimensional atomic chain is considered. As illustrated in Fig. 1a, the atomic chain consists of 28 atoms, including 20 active and 4 fixed ones at two ends. An L-J potential with parameters $\varepsilon_{0}=$ $0.4912 \mathrm{eV}$ and $r_{0}=0.23276 \mathrm{~nm}$ is used to model the interatomic interactions. The cut-off radius for the potential is set to be $0.7 \mathrm{~nm}$; and so 4 atoms are used at the fixed ends with a length greater than the cut-off radius. The initial length $(l)$ of the chain is $7.033 \mathrm{~nm}$ with inter-atomic distance $d=0.2605 \mathrm{~nm}$. Tensile loading is performed in a velocitycontrolled way. Specifically, a velocity of $v_{\mathrm{t}}=l \dot{\varepsilon} / 2$ is imposed to fixed atoms at each end, where $\dot{\varepsilon}$ is the nominal strain rate applied to the chain.

In MD simulations, in order to obtain a stress-free statistical thermal equilibrium state, the initial atomic chain is relaxed for $20 \mathrm{ps}$ at $50 \mathrm{~K}$. Then, an NVE ensemble is used to the active atoms to simulate an adiabatic tensile process. To investigate the rate effect, 20 samples are simulated with tensile strain rates ranging from $4.28 \times 10^{2}$ to $1.00 \times 10^{8} \mathrm{~s}^{-1}$.

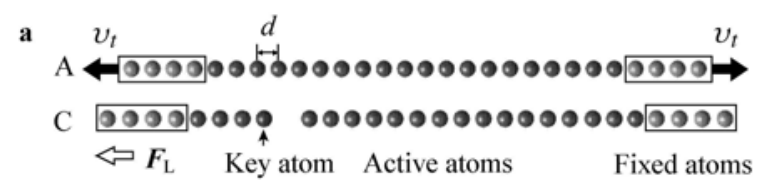

b

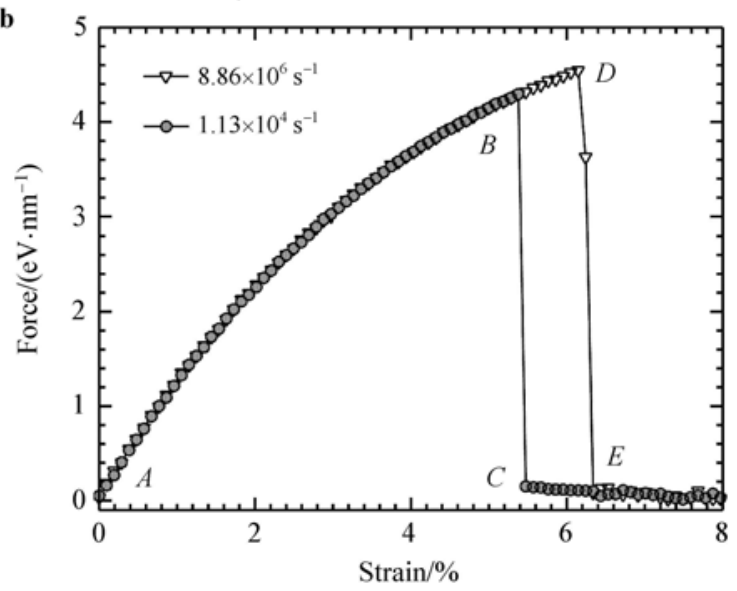

Fig. 1 a Configuration of atomic chain before and after fracture; b Force-strain curves for samples under strain rates of $8.86 \times 10^{6}$ and $1.13 \times 10^{4} \mathrm{~s}^{-1}$, respectively

Physical quantities of the system, for example, the total energy, temperature and so on, are always obtained by means of time-average in MD simulations. This is usually implemented by equilibrating the system for some time (usually several oscillation periods) after a loading step. However in our cases, if the equilibration is a part of the loading process, molecular motions can be significantly affected when the strain rate is very high. So in order to extract statistical quantities, we employed a stand-alone time-average technique. Specifically, to obtain the total energy $E$ at time $t$, the atomic configuration $\boldsymbol{X}_{t}$ is copied to perform the equilibration independently. Similarly, the resultant force on the leftside fixed atoms $\left(\boldsymbol{F}_{\mathrm{L}}\right)$ is averaged for $50 \mathrm{ps}$ to characterize the tensile response of the system. Additionally, as shown later, it would be very hard for MD to simulate the processes under lower rates, like $10^{2} \mathrm{~s}^{-1}$, even for such a small one dimensional system. Thus, we have to develop some new algorithm for the study of rate effects.

Now, we turn to the other extreme of molecular simulations: MST, which is a distinct and efficient numerical approach designed for quasi-static analysis at finite temperature [17]. The core of MST is the particle-oscillator duality, that is, molecules are treated as particles at their equilibrium positions when the mechanical deformation of the molecular system is examined, while they are treated as oscillators with various frequencies when the contribution of their thermal oscillations to deformation is considered. In MST simulations, equilibrium state of a given molecular system can be obtained by minimizing its Helmholtz free energy

$F=\Phi+3 k T \sum_{i=1}^{N} \ln \frac{\hbar\left|D_{i}\right|^{1 / 6}}{k T}$ 
where $\Phi$ is the total potential energy, $k$ is the Boltzmann constant, $\hbar$ is the reduced Plank constant and $D_{i}$ is the local dynamical matrix of molecule $i$. $D_{i}$ can be connected to the oscillator frequencies $\omega_{i}$ in the form of

$|D|=\prod_{i=1}^{3 N} \omega_{i}^{2}$.

Instead of fully tracing the thermal vibrating process as done in MD, temperature effects are taken into account in MST by evaluating the thermal vibrations with local harmonic approximation $[19,22]$. The Helmholtz free energy of a system can be evaluated by substituting $\omega_{i}$ into Eq. (3). By minimizing the free energy, we can obtain the equilibrium configuration of the system, based on which thermodynamic properties can be calculated. It has been shown that MST is very much efficient than MD (about hundred times faster) in various sample sizes $[18,20]$.

Now, one can see that the two extremes of molecular simulations: MD and MST show complementary features. MD is fully dynamic, but limited to a short loading period (or high rate) and very much time consuming; whereas MST is quasi-static, but with some understanding of intrinsic features (frequency of oscillation) and more efficient than MD. Thus, for the study of rate effect, what we can gain from these features?

\section{Coupled evolution of atomic motions and potential landscapes}

In this section, we should extract what we can learn about the mechanisms underlying rate effect from MD and MST simulations.

\subsection{Effect of strain rate on fracture of atomic chains}

We first take a look at what rate effect MD simulations have told us in the given cases. The atomic chain breaks into two parts near a key atom (red sphere in Fig. 1a) when it is stretched to the strain-to-fracture (STF). The resultant forces at the left end $\left(\boldsymbol{F}_{\mathrm{L}}\right)$ for samples under strain rates of $8.86 \times 10^{6}$ and $1.13 \times 10^{4} \mathrm{~s}^{-1}$ are plotted against the tensile strain in Fig. 1b. The STF of the sample under strain rate of $1.13 \times 10^{4} \mathrm{~s}^{-1}$ is $5.46 \%$ which is smaller than $6.23 \%$ of the sample under a higher rate $\left(8.86 \times 10^{6} \mathrm{~s}^{-1}\right)$. That is to say, the strain rate can enhance the chains strength. To investigate how STF is affected by strain rate, samples with various initial velocity distributions are simulated under various strain rates, as shown in Fig. 2. Results show that STF increases by $28.6 \%$ from $5.46 \%$ to $7.02 \%$ as the strain rate ascends from $4.28 \times 10^{2}$ to $1.00 \times 10^{8} \mathrm{~s}^{-1}$. It is noteworthy that strain rate exhibits no detectable effect on the elastic stage (from $A$ to $B$ in Fig. 1b).

3.2 Coupled evolution of thermal frequency and potential landscape

Now, we have to answer why strain rate can dramatically affect STF as shown in Fig. 2. From MST study, we have learnt that thermal oscillation and its variation can greatly affect the behavior of a molecular system. Figure 3 shows the variation of thermal oscillation frequencies and potential landscapes of the key atom during tension. At finite temperature, an atom in a lattice vibrates thermodynamically in its local potential well (Fig. 3b). Strictly speaking, it always vibrates in nonlinear (anharmonic) ways which significantly depend on the configuration of its neighbouring atoms and bonding status. In Fig. 3a, the frequencies obtained with MD simulation and harmonic approximation are plotted against interatomic distance $d$, respectively. MD results should provide real anharmonic frequencies by tracing the trajectory of an atom in its local potential well; while the harmonic frequencies are approximately calculated by square root of the second derivative of the potential well at its bottom. Although the harmonic frequency is just an approximation to the real anharmonic one, it can be calculated more efficiently and very useful for physic analysis, for instance the famous Debye and Einstein hypothesis used for heat capacity analysis.

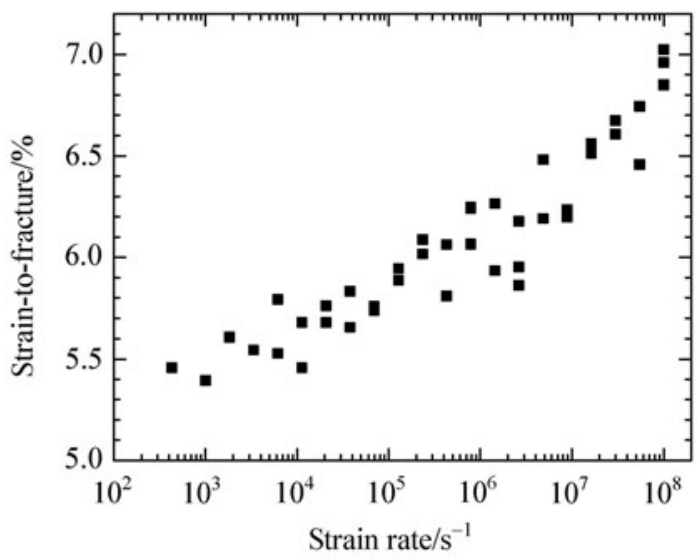

Fig. 2 MD results of strain-to-fracture for atomic chains under tensile strain rates ranging from $4.28 \times 10^{2}$ to $1.00 \times 10^{8} \mathrm{~s}^{-1}$

As shown in Fig. 3a, the atomic frequency obtained with MD reduces nearly to zero at $d=0.305 \mathrm{~nm}$ (point $C$ ) at first and then increases gently. According to the frequency spectrum, we can distinguish three modes of atomic motions in potential wells:

(1) Mode I, as shown in Fig. 3b. When $d$ is smaller than $0.290 \mathrm{~nm}$ (point $B$ in Fig. 3a), the atom vibrates in a potential well with only one bottom. The red dashed line in Fig. $3 \mathrm{~b}$ determined with the atomic kinetic energy indicates the maximum height an atom can reach in a well during thermal vibration. In this case, the harmonic frequencies are very close to the real ones.

(2) Mode II sketched in Fig. 3c. When $d$ falls between points $B$ and $C$, the potential well has split into two bottoms. In this mode, the atom still has enough kinetic energy to climb over the potential barrier. It may oscillate through either of the two bottoms. Since the second derivative of the 
potential landscape at the middle point is minus, the calculated harmonic frequencies become imaginary and therefore non-physical.

(3) Mode III depicted in Fig. 3d. The potential barrier grows up as $d$ increases. At point $C$ in Fig. 3a, the barrier energy becomes the same as the atomic kinetic energy. Beyond point $C$, the kinetic energy becomes too small to cross the barrier. Consequently, the atom can only oscillate near one of the two bottoms (also see the potential well bottom trajectory in Fig. 4). Which bottom the atom will be located in is determined by its initial conditions. Dropping into either of the two bottoms, an atom moves following a mode similar to that of mode I. Most importantly, transition from mode II to III corresponds to the fracture process.
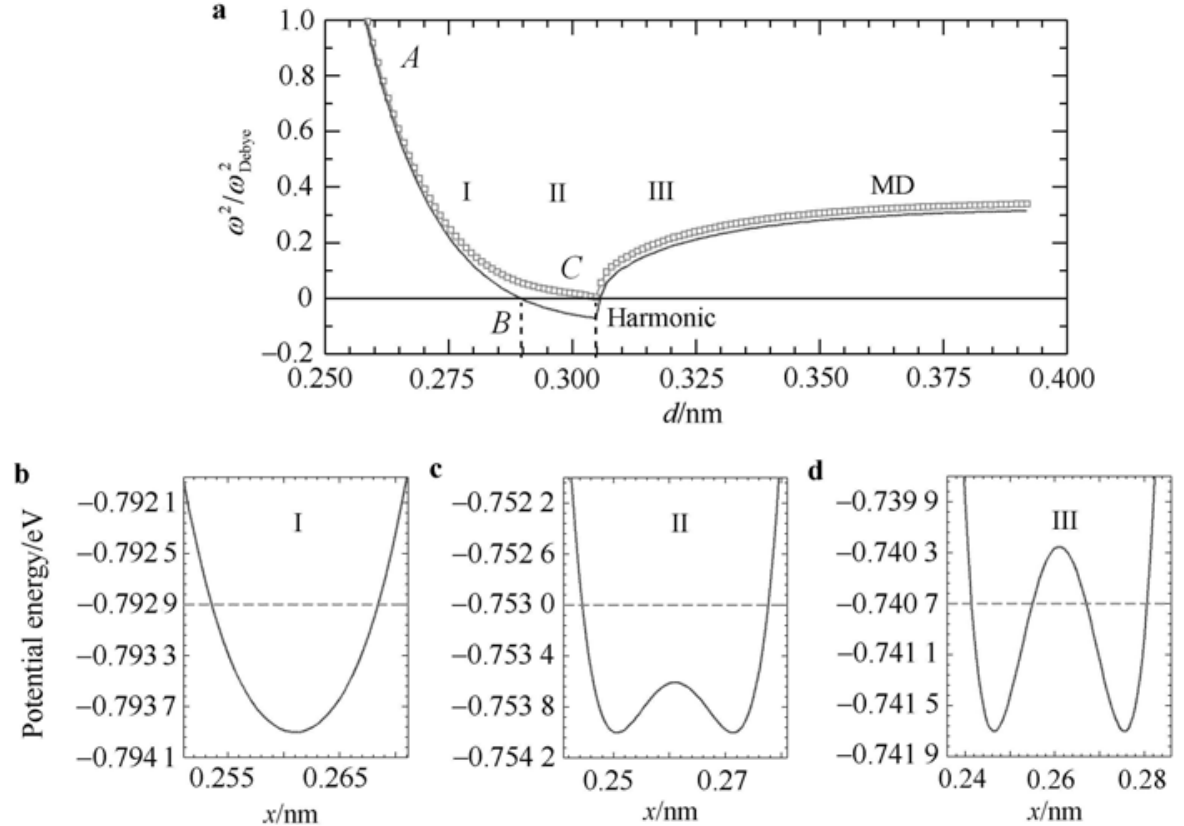

Fig. 3 Atomic frequencies and potential landscapes vary with interatomic distance. a Frequencies (reduced by Debye frequency) calculated by MD and harmonic approximation; b Potential well with one bottom; $\mathbf{c}$ and $\mathbf{d}$ Potential well with two bottoms but different potential barrier. Red dashed lines indicate the kinetic energy

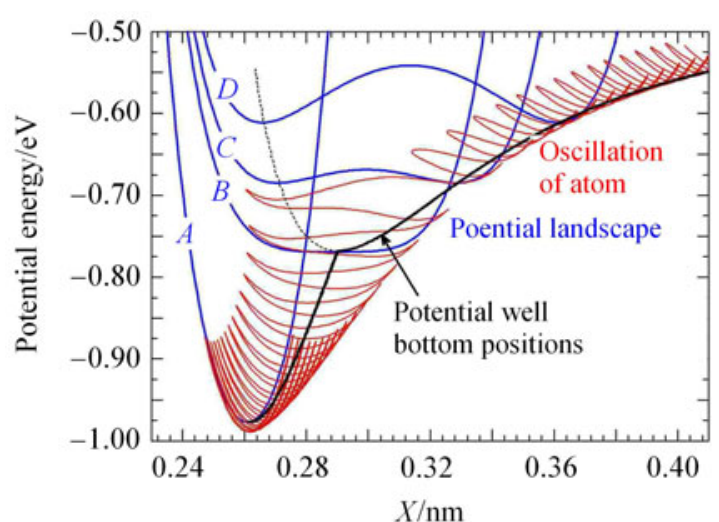

Fig. 4 Oscillation of the key atom (red line), evolution of the atomic local potential landscape (blue line) and potential well bottom (black line) during tensile process

In particular, according to the simulations, rate effect can be traced down to a common microscopic process. Actually, atomic movement in a potential well consists of fast and slow motions, as depicted in Fig. 4. The fast motion refers to the thermal oscillation (closely related to temperature) with an original intrinsic time scale of about $0.1 \mathrm{ps}$, while the slow motion relates to deformation of lattice controlled by external loadings. The time scale involved in slow motion is dependent on loading rate, for example, about $10^{-3} \mathrm{~s}$ in Hopkinson bar tests. Both trajectories of atomic motion and potential well bottoms in the space of energy are plotted against position in Fig. 4 to demonstrate the fast and slow motions. As discussed above, fracture happens when the key atom goes across the potential barrier with its oscillation transforming form mode II to III. During this process, amplitude of the fast motion determines the ability of an atom to across a potential barrier, while the slow motion controls the generation and evolution of potential barriers. However, near the transition point, the rate (or the intrinsic time scales) of fast motion has substantially decreased down to become comparable with that of flow motion, see potential landscape $C$ in Fig. 4 and point $C$ in Fig. 3a. This suggests that the potential field on the right hand of Eq. (2) must be treated as a time dependent term which is closely related to the rate of atomic motions. Therefore, microscopically, the rate effect is governed by the coupled evolution of atomic motions and potential landscapes.

The coupled evolution of atomic motions and potential landscapes can be illustrated as follows. At the begin- 
ning of tension, atoms vibrate in their potential wells with high thermal frequencies $\omega$ (as shown in Fig. $3 \mathrm{a}$ and potential well $A$ in Fig. 4). The intrinsic time scale is the oscillation period $2 \pi / \omega$ which is much smaller than $L / v$ of the loadings time scale. The evolution rate of a potential well is dominated by the tensile loading and is rather slow compared with that of thermal oscillations. As a result, the tensile response shows no rate effect at this stage. With further stretching, the atomic frequency decreases and potential well becomes wide and gentle (as shown in Figs. 3a to $3 \mathrm{c}$ and potential wells $A$ to $C$ in Fig. 4). At a particular strain (point $C$ in Fig. 3a), when the barrier energy of the potential landscape equals to the atoms kinetic energy, atomic frequency tends to zero. This indicates that an atom will oscillate very slowly, especially can be comparable to that of the evolution of potential landscapes. Consequently, the coupled atomic motion and potential landscape would lead to a significant effect on the mechanical properties, for example, STF.

\section{Combined MST/MD simulations of strain rate effect}

Based on the microscopic insight of rate effect, we could develop a new molecular algorithm to more efficiently simulate rate effect and other related mechanical behaviors.

In MD simulations with a time step of $0.01 \mathrm{ps, \text {wecan }}$ trace the whole dynamic processes including thermal motions of each atom. However, it takes several days to simulate the tensile process even for an atomic chain under strain rate of $1.0 \times 10^{3} \mathrm{~s}^{-1}$ on an Intel (R) CPU E6750 core. Hence, MD simulations would be more time-consuming for a real sample under a strain rate close to that in experiments. On the other hand, MST method shows about hundred times higher efficiency than that of MD, but it fails to capture dynamic information of the key atom during fracture. However, before STF most atoms are deep in the potential wells with high frequency oscillations and then the slow deformation process of a sample can still be treated as a quasi-static one. Therefore, we try to combine MD and MST to seek the possibility of developing a new molecular simulation method to balance calculation efficiency and dynamic details.

Considering: (1) strain rate can only affect the fracture process but not the elastic stage; and (2) the key atoms frequency decreases nearly to zero when the atomic chain is close to fracture, MST and MD methods are combined in the following simple way. Firstly, at elastic stage, the atomic chain is stretched using MST. Then we switch simulation from MST to MD for all atoms when the frequency of any active atom is close to zero (in practice, a small trail frequency $\omega_{\mathrm{t}}$ is set with $\omega_{\mathrm{t}}^{2} / \omega_{\text {Debye }}^{2}=0.1$ ). When frequencies of all atoms become greater than $\omega_{\mathrm{t}}, \mathrm{MD}$ is switched back to MST. We call this kind of combination a global MST/MD switching scheme because all atoms, regardless key or normal ones, are simulated using the same approach (MST or MD) before or after switching. Results of global MST/MD scheme are plotted in Fig. 5 and obviously, they are very sim- ilar to that of MD simulations. Moreover, the efficiency of global MST/MD scheme is at least 2 times higher than that of MD.

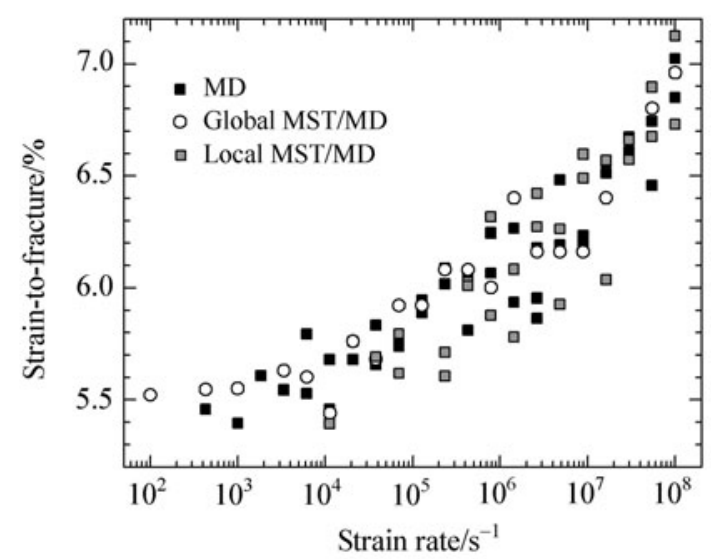

Fig. 5 MD, global MST/MD and local MST/MD scheme results of strain-to-fracture for atomic chains under different tensile strain rates

Normally, only a small part of atoms could be involved into the transition of motion modes during a fracture process, for example the key atoms in atomic chain, atoms near dislocations in crystals, atoms near cracks, etc. Therefore, if we switch MST to MD only for those key atoms during fracture, calculation efficiency may be further improved. This is the idea of local MST/MD switching scheme. The effect of strain rates on STF for this kind of simulations are also exhibited in Fig. 5 and the results are consistent with that of MD and global MST/MD approaches. However, so far, the efficiency of the local MST/MD scheme does not show a prominent improvement over the global one and needs further improvement.

As mentioned above, one goal of this work is to seek alternative and efficient numerical approaches to simulate a molecular system at finite temperature under realistic strain rate and to achieve simulations in consistency with experiments. The results of both global and local combined MST/MD schemes show similar trends of strain rate effect on fracture strain of atomic chains and well agree with that of MD (as shown in Fig. 5). On the efficiency aspect, the global MST/MD scheme is at least 2 times faster than pure MD simulations. The improvement of efficiency is based on the fact that computational consumption of MST is independent on strain rate. On the contrary, computational consumption of MD simulations is inversely proportional to loading strain rates. However, the global MST/MD scheme shows only 2 times of efficiency improvement, because only half of the whole tension process is performed using MST before fracture. Most of the computational consumption is attributed to MD simulation, still with the time step of $0.01 \mathrm{ps}$ for the whole atomic system. Nevertheless, based on these simulation results, it is possible to develop a new algorithm of molecular simulation in future, which could properly demon- 
strate the strain rate effect under the loading rates in laboratory tests. The designed local MST/MD scheme is such one, which is based on the fact that key atoms in a sample are usually localized, like dislocation in a 3D lattice which is essentially a line-defect. This would help us to greatly reduce computational consumption.

\section{Discussions}

As an atomic chain is stretched, three modes of atomic motions can be identified according to variations of atomic frequencies and potential landscapes. In particular, its fracture is a result of the coupled evolution of atomic motions and potential landscapes, which may govern the rate effect on STF. Especially, the fact that the intrinsic time scale of oscillation changes with loading and becomes coupled to external time scale is really extraordinary.

Accordingly, global and local MST/MD combination schemes are developed to simulate molecular systems under a wide range of strain rates. Results of the new schemes are in good consistency with that of MD simulations and with better efficiencies. This verifies the two new algorithms and indicates that rate effect does result from the coupled evolution of atomic motions and potential landscapes.

A more robust local MST/MD scheme is developing underway to efficiently handle molecular systems under realistic strain rates in experiments. The current approaches are expected to be extended to 2 and 3 dimensional systems.

Acknowledgement Computations were performed on the ScGrid of Supercomputing Center, Computer Network Information Center of Chinese Academy of Sciences and the LNM blade of the State Key Laboratory of Nonlinear Mechanics. The paper was presented at the New Models in Hydrodynamics Conference 2012, in London. This conference was organized and supported by Los Alamos National Laboratory (USA), the Atomic Weapons Establishment (UK) and the Institute of Shock Physics (Imperial College London, UK).

\section{References}

1 Armstrong, R.W., Walley, S.M.: High strain rate properties of metals and alloys. International Materials Reviews 53, 105128 (2008)

2 Murr, L.E., Meyers, M.A., Niou, C.S., et al.: Shock-induced deformation twinning in tantalum. Acta Materialia 45, 157175 (1997)

3 Armstrong, R.W., Arnold, W., Zerilli, F.J.: Dislocation mechanics of shock-induced plasticity. Metallurgical and Materials Transactions A 38, 2605-2610 (2007)

4 May, J., Hppel, H.W., Gken, M.: Strain rate sensitivity of ultrafine-grained aluminium processed by severe plastic deformation. Scripta Mater 53, 189-194 (2005)

5 Wang, Y.M., Hamza, A.V., Ma, E.: Temperature-dependent strain rate sensitivity and activation volume of nanocrystalline
Ni. Acta Materialia 54, 2715-2726 (2006)

6 Hoge, K.G., Mukherjee, A.K.: The temperature and strain rate dependence of the flow stress of tantalum. Journal of Materials Science 12, 1666-1672 (1977)

7 Nemat-Nasser, S., Deng, H.: Strain-rate effect on brittle failure in compression. Acta Metallurgica et Materialia 42, 1013-1024 (1994)

8 Suh, N.P., Lee, R.S.: A dislocation model for the delayed yielding phenomenon. Materials Science and Engineering 10, 269278 (1972)

9 Murr, L.E., Trillo, E.A., Pappu, S., et al.: Adiabatic shear bands and examples of their role in severe plastic deformation. Journal of Materials Science 37, 3337-3360 (2002)

10 Murr, L.E., Esquivel, E.V.: Observations of common microstructural issues associated with dynamic deformation phenomena: Twins, microbands, grain size effects, shear bands, and dynamic recrystallization. Journal of Materials Science 39, 1153-1168 (2004)

11 Kadau, K., Lomdahl, P.S., Holian, B.L., et al.: Moleculardynamics study of mechanical deformation in nano-crystalline aluminum. Metallurgical and Materials Transactions A 35, 2719-2723 (2004)

12 Koh, S.J.A., Lee, H.P.: Molecular dynamics simulation of size and strain rate dependent mechanical response of FCC metallic nanowires. Nanotechnology 17, 3451 (2006)

13 Liang, H.Y., Wang, X. X., Wu, H.G., et al.: Molecular dynamics simulation of length scale effects on tension nano crystal line line copper wire. Acta Mechanica Sinica 34, 2 (2002) (in Chinese)

14 Zheng, Z.Z., Bai, Y.L.: Splitting the fast and slow motions in molecular dynamics simulations based on the change of cold potential well bottom. AIP Conference Proceedings 1233, 917$922(2010)$

15 Leach, A.R.: Molecular Modelling. Prentice Hall, USA (2001)

16 Najafabadi, R., Srolovitz, D.J.: Evaluation of the accuracy of the free-energy-minimization method. Physical Review B 52, 9229 (1995)

17 Wang, H.Y., Hu, M., Xia, M.F., et al.: Molecular/cluster statistical thermodynamics methods to simulate quasi-static deformations at finite temperature. International Journal of Solids and Structures 45, 3918-3933 (2008)

18 Xiao, P., Wang, J., Ke, F.J., et al.: Molecular statistical thermodynamics-A distinct and efficient numerical approach to quasi-static analysis of nanomaterials at finite temperature. Composites Part B: Engineering 43, 57-63 (2012)

19 LeSar, R., Najafabadi, R., Srolovitz, D.J.: Finite-temperature defect properties from free-energy minimization. Physical Review Letters 63, 624 (1989)

20 Wang, J., Xiao, P., Zhou, M., et al.: Wurtzite-to-tetragonal structure phase transformation and size effect in $\mathrm{ZnO}$ nanorods. Journal of Applied Physics 107, 023512-023517 (2010)

21 Xiao, P., Wang, X., Wang, J., et al.: Surface transformation and inversion domain boundaries in gallium nitride nanorods. Applied Physics Letters 95, 211907-211903 (2009)

22 Rickman, J.M., Najafabadi, R., Zhao, L., et al.: Finitetemperature properties of perfect crystals and defects from zero-temperature energy minimization. Journal of Physics: Condensed Matter 4, 4923 (1992) 